\title{
LA EDUCACIÓN COSTARRICENSE AL SERVICIO DE LOS GRUPOS DE PODER: UNA VISION DESDE LOS EXAMENES ESTANDARIZADOS, LAS REGULACIONES Y GUIAS DISCIPLINARIAS
}

\section{Vivian Carvajal Jiménez ${ }^{1}$}

\begin{abstract}
Resumen: Muchas de las políticas, estatutos y propuestas que orientan la práctica educativa de nuestro país, tienen su origen en intereses económicos y políticos que nada tienen que ver con el enriquecimiento y desarrollo de los implicados en el proceso escolar. Algunas regulaciones, guías disciplinarias y los exámenes estandarizados, promueven la segregación de ciertos grupos y el mantenimiento del estatus de otros.

En este sentido, está en manos de los docentes la promoción de un modelo divergente que, a pesar de los lineamientos establecidos, estimule la igualdad y motive la inserción de todos los estudiantes al sistema social y productivo del país.
\end{abstract}

Palabras clave: GRUPOS DE PODER / PROGRAMAS DE ESTUDIO / EXÁMENES NACIONALES / ORIENTACIÓN DE LA ENSEÑANZA Y EL APRENDIZAJE

\begin{abstract}
Many of the policies, proposals and statutes, that orientate education in our country, finds their origins in economical and political interests that do not have the enrichment and development of care about those involved in the educational process.

Some regulations, disciplinary guides and standardized exams, promote the segregation of certain groups and maintain the status of others.

So in this sense, it is the teacher's responsibility to proclaim a divergent model before the students instead of or in spite of the should be promote established one equality and the integration of all students to the social and productive system.
\end{abstract}

Key words: SOCIAL CLASSES/ STANDARDIZED TESTS/ DISCIPLINE RULES/ EDUCATION BASIS/

\section{Introducción}

Desde siempre, los sistemas educativos de todo el mundo han sido objeto de severas críticas y constantes cuestionamientos que, a su vez, se revitalizan al ritmo de los descubrimientos, la experimentación de diversos de métodos, y la introducción y desarrollo de la tecnología. A partir de aquí, se han generado preguntas y proyectos que han ampliado el espectro de lo curricular, lo didáctico y lo pedagógico.

\footnotetext{
${ }^{1}$ Magister en Psicopedagogía Licenciada en Ciencias de la Educación con énfasis en Docencia, ambos de la Universidad Estatal a Distancia, Costa Rica. Profesora del Centro de Investigación y Docencia en Educación, Universidad Nacional, Profesora del Liceo Hernán Zamora.
}

Correo electrónico: vivianci@costarricense.cr

Artículo recibido: 16 de enero, 2004

Artículo aprobado: 7 de junio, 2004 
Algunas de las cuestiones más relevantes han sido cómo se aprende, cómo se debe enseñar, qué se debe enseñar y a partir de qué parámetros diseñar el currículo; preguntas básicas en los programas y discusiones generadas en las aulas en que se prepara a los futuros docentes, en las sesiones de capacitación, seminarios, coloquios, cursos, talleres y demás actividades que implican la participación de miembros de la comunidad educativa. El asunto no termina aquí, ni se inscribe solamente a los postulados dictados por reconocidísimos investigadores, incorporados ya a la bibliografía obligatoria de cualquier curso relativo a la Didáctica o el Currículo, como Ausubel, Skinner, Piaget o Vigosky.

Las revoluciones del siglo pasado, los alcances tecnológicos de la era actual, los constantes cambios a que se ven sometidas las sociedades y las transformaciones que fenómenos económicos como la Globalización han generado, suscitan nuevas interrogantes y cuestionamientos: ¿Cómo se debe estructurar un currículo que resulte cultural, temporal y socialmente pertinente? ¿Cómo, en realidad, se equipa a las nuevas generaciones con las destrezas y construcciones que se necesitan para afrontar, en todas sus dimensiones, el mundo moderno?

En esta línea, vale la pena reflexionar en cuanto prácticas, técnicas, estrategias, concepciones contenidos, valores y métodos desarrollados en nuestro sistema educativo: ¿En qué sentido orientan las políticas y acciones educativas implementadas en nuestro sistema? ¿A qué ejes responden los lineamientos dictados por las instituciones regentes? ¿Existe una propuesta curricular institucionalizada? ¿Quiénes determinan qué se debe incluir en el currículo y por qué se estructura de determinada manera? ¿Quiénes se benefician con la orientación pedagógica que se sigue en nuestro país? ¿Al servicio de qué y de quién opera el sistema educativo?

En los párrafos que siguen se desarrolla una tesis que procura, modestamente, generar reflexión en torno a las preguntas planteadas. 


\section{2. ¿Es válido afirmar que los sistemas educativos actúan en función de necesidades políticas o económicas?}

Es por demás sabido que cada individuo posee características, antecedentes y habilidades distintas, y por lo tanto, es un ser único que requiere de diferentes maneras para satisfacer sus muy personales necesidades.

Dichas necesidades no solo se inscriben en la individualidad, sino que tienen estrechas y complicadas relaciones con el medio en que se desenvolvió una persona, con sus antecedentes históricos y familiares, con la época y el país o región donde le tocó vivir, y con un sinfín más de determinantes como la clase social, las convicciones familiares, la etnia, el poder económico, las aspiraciones y la cultura.

En el plano educativo, resulta más que importante tomar en consideración lo anterior, pues todo docente debe ser consciente de que trabaja con personas capaces, diferentes entre sí y pertenecientes a diversas realidades; además, todo buen educador tiene que reconocer que es posible aprender de las experiencias de sus estudiantes, y que aprovecharse de ellas para guiarlos y ayudarlos en la exploración de los conocimientos; ese es su papel fundamental en el salón de clase.

Debido a las difíciles condiciones de trabajo con que topan los educadores en Costa Rica (sobrepoblación estudiantil, malas condiciones en el aula, problemas fuera de la escuela, mala estructura de los planes educativos, imposición de exámenes estatales, etc), tratar de satisfacer las necesidades de cada estudiante, explorar sus potencialidades y atenderlos de manera individual, se vuelve una ardua tarea.

Las nuevas modalidades educativas y los actuales contenidos curriculares contribuyen a que se tenga una visión más amplia del quehacer educativo por parte de estudiantes y maestros. Esto es de vital trascendencia cuando se retoman los propósitos que sostienen lo que debería ser, a todas luces, el propósito universal de la Educación: generar igualdad.

En el caso de la Educación Costarricense, este 2004 arranca con una encomiable propuesta: Relanzar la Educación; esto es, innovar educando a todas y todos, y propiciando auténticas y equitativas oportunidades de inclusión escolar. Como parte de ello, se trabaja 
fuertemente en el desarrollo de adecuaciones curriculares de calidad, que no se centren en retirar un ítem de las pruebas, sino en favorecer las destrezas y estilos de aprendizaje de los estudiantes en el desenvolvimiento mismo de la práctica pedagógica. Además, se comienzan a dedicar capítulos de actualización y capacitación para docentes en ejercicio, en los que se aborda la temática de la transversalidad: importantes ejes que estimulan a la reflexión, la crítica, el reconocimiento de lo autóctono y la proyección de valores.

En relación con lo anterior, Sciacca (1976, p.112), refiriéndose a los propósitos que cualquier programa educativo debería contemplar, sostiene que:

. . . se trata de crear individuos libres y responsables y no títeres de las modas, intereses e ideologías dominantes; se trata de formar hombres cultos, o sea, capaces de recrear interiormente y revivir los contenidos que la escuela propone, y no repetidores hoy, tal vez de unos clásicos mal digeridos o de unas nociones científicas aprendidas de memoria, y mañana, de unos 'slogans' cualquiera . . . buscamos los medios para formar la sede de la interioridad, o sea, la conciencia y su autonomía. Sin ella, no hay 'humanidades' ni Humanidad.

No obstante, a la hora de desarrollar un proyecto que busque la génesis de individuos libres, hay que considerar algunos aspectos vitales del ámbito educacional; en especial, cuando se han institucionalizado toda una serie de normas que bien pudieran actuar como armas de doble filo: por un lado, porque ofrecen parámetros que permiten medir algunas características y obtener información que sirva de sustento a ciertas decisiones o prácticas; por otro, se trata de prácticas que no garantizan el aprendizaje real, y en un sentido, pueden incluso interferir con la adquisición y el disfrute genuinos (concentración de energía en los resultados y no en los procesos). Ejemplo de ello, las pruebas estandarizadas, aplicadas a todos los estudiantes del sistema, independientemente de su condición, contexto, intereses o habilidades.

Por otra parte, en vista de las diferencias que distinguen a los grupos de estudiantes que asisten a las distintas modalidades educativas, es importante y necesario hacer una revisión de la metodología, la motivación y el seguimiento que se emplea en las aulas para, consecuentemente, valorar su efectividad y hacer las modificaciones que se requieran, no 
solo a nivel micro, sino también desde el ente encargado de manejar todo lo relativo a la educación costarricense.

Tomando en consideración lo anterior, se puede decir que no se trata simplemente de realizar observaciones ni poner sobre la mesa las divergencias existentes entre las estrategias metodológicas que se utilizan; más bien, se deben identificar problemas, ofrecer sugerencias y abrir una puerta para que se emprenda la tarea de corregir a profundidad lo que sea necesario; para que se pongan en marcha planes y soluciones, y para llenar los vacíos que pudieran estar afectando la eficiencia educativa de la enseñanza institucional dirigida a costarricenses; en palabras de Bernardini (1985, p.31):

Para mejorar la educación costarricense hay que revisar muy a fondo la formación de los educadores; profesionalizarlos, es cierto, pero no se puede creer que el título universitario solucione todos los problemas. Habría entonces que revisar la mentalidad, los criterios formativos que están detrás de los títulos.

Si se quiere ofrecer un panorama general en cuanto a los puntos que necesitan mejoras en la enseñanza, primero debe revisarse la estrategia metodológica que los educadores emplean y los postulados que aparecen en los programas y que el Ministerio de Educación Pública da como rubros a seguir. No se puede obviar el hecho de que los rubros y condicionamientos que son impuestos desde "arriba", obstaculizan muchas veces la labor de los docentes bienintencionados, de facilitadores creativos y experienciales que además del sistema, deben luchar contra la tradición practicada por muchos de sus colegas. Así como menciona Guerrero, (citado pro Ortega 1989, p. 16) el reto que la educación plantea es: ". . ¿ ¿cómo hacer para que no sea una disciplina estancada, desconvexa y asilada, sino, antes al contrario, interrelacionada con otras?".

Hallar una solución a este problema resulta imprescindible, pero hacerlo, implica al mismo tiempo, una labor titánica que no todas las sociedades están dispuestas a emprender, no solo por el trabajo que en sí demanda, sino porque implicaría una inversión de tiempo y energía sobresaliente, sin hablar del acomodo de las estructuras y mentalidades que eso necesariamente conllevaría, pues, como expone Marx (citado por Ortega, 1989, p. 19) ". . . las clases dominantes desarrollan formas ideológicas que legitiman su dominación: la clase que tiene los medios de producción material a su disposición, tiene al mismo tiempo 
el control sobre la producción cultural", y por ende, sobre los mecanismos que llevan esta a las masas, la más elemental por supuesto, la escolarización.

En el caso de Costa Rica, claramente se observan tendencias que legitiman intereses de clases económicas poderosas y de grupos interesados en el desarrollo de capital y mano de obra. Por ejemplo, se avecina el peligro de condicionar las clases de Arte, Educación para el Hogar y Artes Industriales, materias clave en el desarrollo de destrezas motoras, el descubrimiento de talentos y el desarrollo de una educación integral y humanista.

La idea de que una buena educación se basa únicamente en dominar el inglés (aunque las metodologías aplicadas no generan personas bilingües al cabo de cinco años de "estudiar" el idioma), saber manejar una computadora y navegar por Internet, es una concepción muy pobre, que vislumbra grandes cantidades de mano de obra barata: personas que con dificultad pueden leer y escribir, analfabetos de la historia y cultura latinoamericana, ignorantes de las producciones y manifestaciones artísticas . . . Lamentablemente, esta idea se populariza entre las masas, e incluso, algunos docentes, supuestamente partidarios de la educación integral, se dejan decir que las llamadas asignaturas especiales, no aportan nada significativo al currículo.

Berstein (citado por Ortega, 1989, p.21) señala que " . . .la forma en que una sociedad clasifica, distribuye, transmite y evalúa el conocimiento educativo que considera como público, refleja a la vez la distribución del poder y los principios de control de la sociedad". Siendo esto así, cabe preguntarse el papel que desempeñan las prácticas pedagógicas generadas en el aula, los contenidos administrados y la manera en que estos se evalúan, sumativamente, al final de cada trimestre y en las pruebas nacionales; así como la idea de "educación de calidad" que los propios docentes, y el sistema en general, les transmiten a padres de familia y estudiantes..

Asimismo, la reflexión en torno a porqué se fragmentan temáticas y áreas del conocimiento como norma instruccional, y el hecho de que se centre más la atención en aspectos disciplinarios que en propuestas de aprendizaje, debería estimular la reflexión en torno a qué se persigue y qué se consigue en realidad en nuestros salones de clase: ¿Se está estimulando la creación de individuos independientes, críticos, creativos e innovadores? Al respecto, una alusión de Varela, quien asegura que (1984, p.13) la escuela 
funciona más como un ente controlador, como un "encierro" donde se condiciona a las futuras generaciones y se les instruye de acuerdo con la política dominante, ". . . es la institución donde se ejerce la violencia destinada a perpetuar el poder mediante la representación de legitimidad de un arbitrario cultural . . ."; es en esta línea que la escuela inculca hábitos y leyes de sumisión y obediencia que responden a visiones ideológicas y disciplinarias.

Para Boudon, citado por Ortega (1989, p.27), la escuela es ". . . una cortina de humo o un espejismo destinado a hacer esperar lo irrealizable, desviando la atención del problema central que es la reducción de las desigualdades". Por lo anterior, resulta por demás curioso ver cómo los docentes, y las instituciones en general, concentran más sus energías en verificar que todos los estudiantes anden con medias azules que en interesarse por promover la investigación, la participación, la crítica, el análisis, la reflexión y la vivencia de conocimientos aplicables y valiosos. Este ejemplo correspondería con lo que Foucault (citado por Ortega, 1989, p.33) señala como una manifestación de ". . . una institución con carácter represor de encierro", hablando de la cuarentena a la que se ven sometidos los niños, según él, al igual que los locos, los pobres y las prostitutas. La institución escolar, en ocasiones, más que contribuir a la superación económica y a la disminución de las desigualdades (como promueven los políticos en su campaña), puede ser una estructura que colabore en el mantenimiento y consolidación del poder dominante, con lo que necesariamente la estratificación y jerarquía social y económica se mantienen. Esto es, permitiendo, y a veces promoviendo, la deserción escolar (muchas veces, el sistema expulsa a quien no se ajusta, y en pocas oportunidades, realmente, se ofrecen alternativas viables o ayudas legítimas a quines más lo necesitan: los estudiantes cuyas NEE se dan por su condición socioeconómica o cultural).

Como relata Varela (1984, p. 53), en la escuela

". . .el tiempo se convertirá en un tiempo útil, en el que no habrá lugar para las distracciones ni para las actividades que pongan en peligro la marcha de la clase ... las materias graduadas deben ser atravesadas sin retrasos si se quiere alcanzar exitosamente la meta. . . . Exámenes, ejercicios y pruebas, cobran toda su significación en el interior de este tiempo y espacio disciplinarios, a la vez que 
permiten ocultar los poderes concretos que se ejercen en nombre del saber pedagógico".

En este sentido se pueden situar, nuevamente, algunas prácticas pedagógicas que se siguen en Costa Rica, pues no solo se estructuran los planeamientos y objetivos en función de completar contenidos, independientemente de cómo se haga ni de la vigencia o importancia de los mismos, sino que se asegura su terminación con exámenes al final de cada una de las etapas de la Educación General Básica y el Ciclo Diversificado; poniendo así a toda carrera a estudiantes y maestros, pues el fin no es aprender ni experimentar ni enriquecerse ni crecer, sino abarcar la materia, memorizarla por un rato y pasar los exámenes.

Adicionalmente, por la premura de las Pruebas Nacionales, los Programas Educativos difícilmente pueden variarse, no solo porque se va contra reloj (los contenidos son extensísimos), sino porque la mayoría de profesores ya se ha institucionalizado y acostumbrado a limitarse y funcionar al mínimo, lo que dificulta cualquier intento de cambio. A esto, debe añadirse que los contenidos de las distintas materias no están, en absoluto, articulados o integrados. Así, mientras en literatura se está estudiando la mitología helénica, en Estudios Sociales, en lugar de ver el legado de la cultura griega, se está estudiando la geografía de Costa Rica.

Los contenidos mismos de los Programas son cuestionables, y si no se está seguro de ello, basta con echarle una mirada al programa de estudio de Español, según el cual, los alumnos de octavo año (que en promedio tienen 14 años de edad) deben leer La Vorágine, una novela cuya dificultad analítica exige un nivel de abstracción que no se alcanza sino alrededor de los 17 años; o, tomando por caso un tema, ¿de qué le sirve a un adolescente reconocer una frase subordinada adjetiva o sustantiva? ¿No sería mucho más productivo e interesante ayudarlo a descubrir los mecanismos del discurso ideológico dominante, analizar e interpretar por su cuenta textos (llámese caricatura, canción, periódico, fotografía, programa televisivo, etc) que nos bombardean a diario y que pretenden manipularnos o inducirnos a hacer o aceptar $\mathrm{X}$ cosa? Por supuesto, pero esto no está en concordancia con las disposiciones de las clases dominantes, entendiendo estas como todos aquellos grupos elitistas, propietarios de la riqueza y manipuladores del poder adquisitivo, político o cultural del país. 
Al respecto, vale la pena retomar las palabras de Varela (1984, p. 66), quien enuncia:

La infancia popular ha de ser domesticada, y ¿qué medio mejor para regenerarla que la ciencia pedagógica que, además de sustraerla de los peligros y enseñarle buenas costumbres, hace penetrar a la civilización en el hogar ayudando a desterrar todo tipo de vicios de las clases trabajadoras, percibidas por la burguesía como clases ignorantes, inmorales y peligrosas?

¿Será este uno de los objetivos de la educación "gratuita y obligatoria"? ¿Se busca en realidad adiestrar a las clases dominadas para seguirlas dominando? En un sentido, podría ser. A manera de ilustración, valga mencionar que, en la mayoría de regiones costarricenses, el docente apreciado y reconocido por padres y colegas es aquel que mantiene a sus niños como estatuas mientras copian y transcriben lo que ya está en su libro de texto; permitiéndoles así memorizar hechos ya verificados para pasarlos luego al examen, y por lo mismo, 'salir bien' en las pruebas. Si el asunto es siempre así, es comprensible la afirmación que hace Ortega (1989, p.70), cuando asegura que ". . .la escuela no produce cualificación profesional, sino que aparca a menores y jóvenes que de otra manera podrían resultar peligrosos para el orden social". Dichosamente, a la par de esta realidad, cientos de educadores trabajan incansablemente por realizar inclusiones educativas de calidad, aun en medio de difíciles obstáculos económicos que, como país en vías de desarrollo, resultan inevitables.

Las ideas de adoctrinamiento que dieron origen a los sistemas educativos continúan, de alguna manera, permeando e influyendo el currículo que se sigue en las instituciones educativas costarricenses. Ciertas prácticas, lejos de acuñar el concepto de Brunner (1999, p. 108), según el cual la educación debe verse como ". . . un proceso interactivo en el que las personas aprenden unas de otras", el sistema costarricense, muy a pesar de los esfuerzos de unos pocos, y muy a pesar de contar, por fin, con programas que incluyen la transversalidad y sugieren una aplicación práctica y constructiva de los procedimientos, sigue perpetuando valores tradicionalistas y verticales, supeditados a los intereses de ciertos grupos, la elite clasista y las prioridades económicas de unos pocos. Estos grupos, llamados en este artículo "dominantes", están representados por la burguesía, los políticos acaudalados ligados a la Iglesia, y las enormes compañías transnacionales que echan raíces y cosechan intereses entre quienes se corrompen por interés personal 


\section{3. ¿Para qué se educa?}

Cada vez que se visita un aula de clase costarricense y se observa la práctica docente que se desarrolla, queda la sensación de que, pese a todas la ideas curriculares que las universidades promueven, y de que los estudiantes de docencia aseguran estar concientizados respecto de las técnicas participativas y experienciales, hace falta algo, o hay algo de más que no permite desarrollar lecciones amenas y productivas.

Creer o dejar de creer en la buena voluntad de los maestros no es la cuestión. Es que se hace cada vez más necesaria la revisión profunda, articulada y minuciosa de las verdaderas motivaciones que han impulsado la creación de programas educativos, exámenes estandarizados y prácticas ideológicas que transmiten valores, conductas e ideas.

Nuestra educación es, sin lugar a duda, un elemento social que reclama un cambio integral. Esto por supuesto no se logra de la noche a la mañana, ni es posible sin que haya un costo de por medio, pues ". . . la introducción de una innovación en la enseñanza necesariamente implicará cambiar las teorías psicológicas y pedagógicas populares de alumnos y maestros. En una palabra, la enseñanza está inevitablemente basada en nociones sobre la naturaleza de la mente del aprendiz" (Bruner, 1990, p. 77).

Al hablar sobre estas nociones, cabe preguntarse si las que se imponen en el ámbito educativo costarricense corresponden o no, en alguna manera, con intereses externos que quieren concebir a los estudiantes como receptáculos que están abiertos a aceptar las "verdades" de los grupos dominantes, las económicas más poderosas, la elite, la burguesía y los dueños de las grandes compañías que se instalan en el país. ¿De dónde surgen y por qué se mantienen ideas que marcan a la gente? ¿Por qué algunas prácticas se aseguran de que los pobres lo sigan siendo (falta de acceso educativo genuino para grupos marginados, por ejemplo) y de que el razonamiento popular de que "el que nació pa'maceta del corredor no pasa" se conserve, construyendo de paso un concepto de "maceta" que envuelve a un buen grueso de la población: aquellos que se constituyen en la mano de obra barata que trabaja en las transnacionales y fábricas donde tienen alguna inversión los políticos o líderes religiosos al mando? Así lo manifiesta Gardner (2000, p. 24): "Nuestra concepción de la escuela está estrechamente vinculada con la existencia de sistemas escritos formales cuyo dominio se ha considerado necesario por motivos económicos, religiosos o sociales". 
¿Para quién entonces trabajan los docentes? ¿Es en realidad para colaborar como orientadores en el proceso formal de aprendizaje por el que deben atravesar todos los niños y niñas de nuestro país? ¿Es para apoyar los mecanismos de segregación de clases? ¿Es para manifestar que se apoya la manipulación abierta y desmedida que se hace de los costarricenses? Ya la corriente credentista había manifestado que ". . . la escuela era simplemente un lugar donde adquirir títulos que luego serían utilizados por los individuos y grupos como un instrumento legítimo en la pugna por las ventajas relativas en la vida adulta" (Fernández, citado por Ortega, 1989, p.28).

Siempre sobre esta ruta, se encuentra además un proceso al que se han visto sometidos los países en vías de desarrollo: la globalización. El sistema educativo no escapa a esa tendencia; por el contrario, es la institución ideal para calmar a la masa y hacerle creer al mundo que Costa Rica es una nación "civilizada" y actualizada, hábilmente incorporada a los mercados mundialistas; y para ello, urge calmar al pueblo haciéndole pensar que se está llevando tecnología e idiomas a las aulas de clase.

Al darle una mirada a los programas de Inglés y Francés que se imparten en escuelas y colegios, al tipo de metodología que se aplica en estas lecciones y al absurdo de decirle a la gente que se está adquiriendo una segunda lengua porque se puede saludar en otro idioma o porque se saben de memoria algunas frases, queda claro que el objetivo primordial, en realidad, no se centra en generar una sociedad que se interese por la diversidad, ni una generación inclinada al conocimiento y comprensión de lenguas y culturas.

La globalización le exige a los grupos de poder que la población "masculle alguito de inglés y sepa un poquitico de computación"; de esta manera, casi cualquier tico joven podrá emplearse en las grandes transnacionales (donde llegan extranjeros que solo entienden inglés y se usan las computadoras para hacer pedidos y sellar facturas), en los hoteles turísticos (cuyos propietarios son, generalmente, extranjeros adinerados) y en uno que otro negocillo (a lo mejor del sobrino de alguna figura pública). Todo esto, de paso, por salarios mínimos - de hambre, garantías a medias y la imposibilidad de exigir más porque la oferta es más alta que la demanda. 
Por lo tanto, no sorprende el que las palabras de Gardner (2000, p. 146) se cumplan, en algún sentido, en el caso de las instituciones educativas costarricenses, pues " . . . transmiten material en un contexto que suele ser ajeno a aquél donde ese material se acabará aplicando. La escuela es, en gran medida, un marco descontextualizado". Lo importante en realidad es que ". . . se tienen que estudiar, memorizar, emplear cuando sea oportuno y transmitir a las siguientes generaciones los cuerpos doctrinales", sean técnicas que le permitan a los poderosos contar con mano de obra capacitada y económica, o elementos que le hagan sentir al pueblo que se le está reconociendo su derecho a la educación.

Elizalde (1991, p.62) manifiesta su desacuerdo con las posiciones globalizantes que han sido implantadas en el quehacer educativo al decir que ". . . nuestra educación homogeniza conocimientos, habilidades, saberes, destrezas, aptitudes, capacidades . . Con esto, ella tiende a castrar todas las diferencia que existen entre los distintos individuos. ¿No es esta una forma de amputación social?" Tan enraizada está esta idea en las mentes de los participantes de los sistemas educativos, que a veces, ni siquiera se puede comprender la diferencia en los resultados tras la aplicación de los mismos métodos con grupos distintos.

¿Qué pueden hacer los docentes si los lineamientos que rigen su práctica pedagógica vienen de "arriba"? En primer lugar, es necesario que los maestros dejen de ver a sus estudiantes como recipientes sin experiencias ni pensamientos, que dejen de adiestrarlos en la copia y memorización textual y los alienten a lanzarse a experiencias positivas, analíticas e investigativas. No es fácil, pues de hecho, hasta los mismos alumnos ya están moldeados al sistema de "dicte, copie y memorice por un rato", pero solo haciendo cambios en la práctica docente se podrá intentar pelear por conseguir cambios de fondo que incluyan las estipulaciones ministeriales.

Transformar prácticas sustentadas en ideologías que la institucionalización ha consolidado es sumamente difícil, pero al menos se podrá identificar aspectos y técnicas que contribuyan a un mejoramiento integral de la población.

\section{4. ¿Cómo se aprende?}

Como ya se ha dicho, los programas educativos que se imparten en las aulas de nuestro país, no solo se sustentan en las capacidades, aptitudes, experiencias y 
necesidades de los estudiantes, sino que, en amplio margen, se fundamentan en una práctica tradicional y conservadora en la que privan los intereses de los grupos de poder. Al mismo tiempo, las condiciones económicas y la mala distribución de los recursos, hacen que los salones de clases sean verdaderos centros de batalla para docentes y alumnos.

Las posiciones epistemológicas de tipo constructivista y cognoscitivista, promovidas por algunos docentes en las aulas universitarias, rara vez se llevan a la práctica en la realidad cotidiana de nuestros centros de enseñanza, ya que asumir una posición creadora e innovadora requiere mucho más que el manejo de unos postulados teóricos; requiere de estimulación, planificación, destrezas, y motivación al pensamiento divergente; dado que

. . semejante posición filosófica implica, en primer lugar, que el conocimiento humano no se recibe pasivamente ni del mundo ni de nadie, sino que es procesado y construido activamente por el sujeto que conoce. En segundo lugar, la función cognoscitiva está al servicio de la vida, es una función adaptativa, y en consecuencia lo que permite el conocimiento al conocedor es organizar su mundo experiencial y vivencial (Flores, 1994, p.235).

Si se comparte una visión que contemple el aprendizaje como construcción, entonces, es imprescindible que todo educador sea consciente de su papel como guía y orientador de procesos enriquecedores, dinámicos y empapados de experiencias que le faciliten a cada participante un cúmulo de acciones vívidas y ricas en participación, análisis, reflexión y producción; ya que, “. . . la enseñanza constructivista considera que el aprendizaje humano es siempre una construcción interior" (Flores, 1994, p. 237); en otras palabras, se trata de elaboraciones individuales permeadas por su contexto social, imposibles de ser contempladas y examinadas por una sola lupa, ni desde un solo ángulo.

Independientemente de los obstáculos que invaden el ámbito educativo, llámese programas, exámenes de Bachillerato, literatura o contenidos que no son apropiados ni atractivos a la edad de los estudiantes; es indispensable que el educador retome su compromiso de servicio y entrega, y que se proponga, aún a pesar de las múltiples barreras que entorpecen un buen desenvolvimiento de los estudiantes, crear un ambiente de clase favorable y creador, que, como citaba Flores, propicie construcciones personales, únicas, efectivas y duraderas. 
Por ello, es llamativo el hecho de que la Educación como sistema, pretenda generalizar ideologías, contenidos, conductas y contextos (todo según la "nota" globalizadora), cuando estos van dirigidos a niños y niñas de todos los estratos sociales, criados en las diversas realidades ambientales, económicas y culturales del país: a chiquitos de áreas rurales por igual que a los más citadinos, a los niños de La Carpio y a los de Los Yoses, a los niños trabajadores y a los que van de vacaciones a Miami o al Barceló, a los que juegan con llantas viejas, con play station o a los que ni siquiera juegan.

Nuestro sistema educativo uniforma, aunque la realidad es diversa. Es el papel del docente adecuar hasta donde pueda, adaptar a las circunstancias y hacer de la experiencia algo positivo. Es verdad que existen muchas cosas en contra, es verdad que la orientación de nuestro sistema es, en ocasiones, elitista y segregadora; pero también es verdad que las y los educadores pueden hacer mucho para colmar de crecimiento y aprendizaje sus salones de clase.

Definitivamente, uno de los principios básicos de todo sistema educativo debería ser la sensibilización hacia las diferencias sociales, económicas y políticas que existen en el país, pues la concepción que se tenga acerca de estos aspectos, así como los prejuicios o preferencias, incidirán directamente sobre el proceso de enseñanza-aprendizaje. Como señalan muchos de los grandes promotores del Cognoscitivismo, los pares juegan un papel relevante en todo este proceso, y es el sistema mismo, y el docente como guía, quien debe asegurarse de que esa experiencia sea productiva; es decir, que esté libre de burla, crítica mal sana y prejuicios.

Hasta ahora todo lo que se ha mencionado en pro de formar una educación reflexiva y divergente, se inclina hacia una estrategia pedagógica que convierta las aulas en auténticos laboratorios de construcción del conocimiento. Para eso, cada docente debe ver, en cada uno de sus niños y niñas, un caudal de información, vivencias y conocimientos que requieren ser socializados a través de técnicas que los empujen a compartir, expresarse y opinar, en medio de un ambiente de respeto, solidaridad y participación, y no en una "jaula" en la que se deben pasar 6 horas diarias copiando y transcribiendo. 
Así que, todos, maestros, profesores, padres, cualquiera que sea la relación que ponga a alguien en el papel de guía de las nuevas generaciones, deben aprovechar las oportunidades y hacer la experiencia educacional sea en realidad constructiva y beneficiosa.

\section{A manera de Conclusión}

A pesar de que ciertas políticas o algunos de los recursos sean manejados en beneficio de unos pocos con el deseo mantener la estratificación social y el poder, o con el fin de uniformar y obviar una realidad que desborda en divergencias; todo educador, si está consciente del hecho, puede servir como promotor de una actitud reflexiva, integradora y creadora, desde el salón de clase, y puede transmitir acciones que marquen la diferencia; tal vez, estableciendo el impulso que permita que algunos de los supuestamente excluidos alcancen éxito dentro del sistema.

Desde luego, innovar y promover una inclusión educativa real y de calidad es la única manera de sentar las bases para una transformación auténtica, eficaz y pertinente, en que sean niñas y niños, jóvenes y adultos en formación permanente, los genuinos beneficiados.

\section{Referencias}

Asociación Nacional de Fomento Económico. El Modelo Educativo Costarricense. (1985) San José: Editorial ANFE.

Brunner, Jerome. (1999). "Cultura, mente y educación" en La educación puerta de la cultura. Madrid: VISOR.

Carretero, Mario (1998). Introducción a la Psicología Cognitiva. Buenos Aire: Editorial AIQUE.

Elizalde, Antonio (1991). Cambios de paradigma, educación y crisis: pasos hacia una epistemología integradora y participativa. ¿Superando la racionalidad instrumental?. Programa Educación y Derechos humanos.

Flores Ocho, Raquel (1994). Hacia una pedagogía del conocimiento. Bogotá: McGraw Hill Interamericana, S.A.

Gardner, Howard. (2000). La educación de la mente y el conocimiento de las disciplinas. Barcelona: Paidós.

Ortega, Félix y otros (1989) Manual de Sociología de la Educación. Madrid: VISOR.

Varela, J. (1984). Modos de Educación en la España de la Contrarreforma. Madrid: Editorial La Piqueta. 